\title{
Perfil do uso de Medicamentos entre Estudantes do Ensino Médio de uma Escola Pública do Rio de Janeiro
}

\author{
Thales Brandi Ramos, ${ }^{1}$ Selma Rodrigues de Castilho ${ }^{2}$
}

\section{RESUMO}

Os adolescentes formam um grupo alvo de frequente ação publicitária e alta prevalência de automedicação. O presente trabalho tem como objetivo traçar o perfil do uso de medicamentos por alunos do Ensino Médio de uma escola pública do Rio de Janeiro. Foi realizado um estudo transversal com questionário semiestruturado envolvendo o uso de medicamentos. Os resultados indicam que $31,90 \%$ dos estudantes fizeram uso regular, enquanto 77,16\% usaram algum medicamento nos 15 dias anteriores à pesquisa. Os medicamentos mais citados foram dipirona, Dorflex ${ }^{\circledR}$ e anticoncepcionais. O uso de medicamentos esteve relacionado ao gênero feminino, consultas médicas no último ano e a outros profissionais de saúde. O uso nos 15 dias anteriores também esteve relacionado à automedicação, prática cuja frequência regular foi relatada por $17,67 \%$ dos estudantes, principalmente para alívio de dores (63,09\%), alergia (17,35\%) e alívio de tensões e estresse (10,41\%). O elevado percentual de uso de medicamentos e automedicação reforça a relevância da educação em saúde e da promoção do uso racional de medicamentos nas escolas.

Palavras-chave: Medicamentos; adolescentes; escola; saúde; automedicação.

\section{DRUG USE PROFILE AMONG HIGH SCHOOL STUDENTS OF A PUBLIC SCHOOL IN RIO DE JANEIRO}

\section{ABSTRACT}

Adolescents make a target group for frequent advertising and a high prevalence of self-medication. This paper aims to outline the profile of medication use by high school students in a public school in Rio de Janeiro. A cross-sectional study was carried out with a semi-structured questionnaire regarding the use of medications. The results show that $31.90 \%$ of the students used it regularly, while $77.16 \%$ used some medication in the 15 days prior to the research, with the most mentioned drugs being dipyrone, Dorflex ${ }^{\circledR}$ and contraceptives. The use of medicines was related to the female gender, medical appointments in the last year and to other healthcare professionals. The use in the previous 15 days was also related to self-medication, a practice whose regular frequency was reported by $17.67 \%$ of students mainly for pain relief (63.09\%), allergy (17.35\%) and tension and stress relief (10.41\%). The high percentage of medication use and self-medication reinforce the relevance of health education and the promotion of rational drug use in schools.

Keywords: Drugs; adolescents; high school; health; self-medication.

RECEBIDO EM: 15/6/2020

MODIFICAÇÕES SOLICITADAS EM: 7/3/2021

ACEITO EM: 7/7/2021

\footnotetext{
${ }^{1}$ Autor correspondente. Faculdade de Farmácia - Universidade Federal Fluminense. R. Dr. Mario Vianna, 523 - Santa Rosa. Niterói/RJ, Brasil. CEP 24241-000. http://lattes.cnpq.br/5501961270763349. http://orcid.org/0000-0002-1442-1020. thalesbrandi980@gmail.com

2 Universidade Federal Fluminense. Niterói/RJ, Brasil.
} 


\section{INTRODUÇÃO}

Adolescentes representam o grupo com maior disposição ao uso inadequado e sem controle médico de medicamentos. No Brasil, de acordo com o Sistema Nacional de Informações Tóxico-Farmacológicas, 25,18\% das intoxicações estão relacionadas ao uso de medicamentos, sendo $17,4 \%$ dos casos na população entre 10 e 19 anos de idade, ${ }^{1}$ tornando imperativo que haja melhoria na racionalidade do uso de medicamentos nesta população.

O uso de medicamentos entre adolescentes ainda não é bem discutido na literatura, especialmente em países em desenvolvimento. A maior parte dos estudos que avalia o consumo de medicamentos para essa faixa etária foca suas análises em questões sociodemográficas e socioeconômicas, além de buscar estabelecer o perfil de uso de medicamentos, automedicação, visitas ao serviço de saúde e indicação para uso de medicamentos. ${ }^{2-5}$

Um estudo recente e relevante para entender o padrão de utilização de medicamentos no Brasil foi a Pesquisa Nacional sobre Acesso, Utilização e Promoção do Uso Racional de Medicamentos (Pnaum), ${ }^{6}$ que mostrou que 30,6\% da população entre 10 e 19 anos fazia uso de medicamentos, com maior consumo para doenças agudas do que crônicas (24,6\% contra 5,3\%). A proposta do Ministério da Saúde era utilizar esses dados para estabelecer indicadores nacionais sobre acesso e uso racional de medicamentos, com o intuito de priorizar os rumos estratégicos de políticas e programas. ${ }^{7}$

Desta forma, torna-se imperativo que o uso inadequado de medicamentos possa ser minimizado tanto por políticas regulatórias quanto por ações de educação e informação da população. Nesse sentido, a Organização Mundial da Saúde (OMS), com o apoio da Organização Pan-Americana de Saúde (Opas), elaborou um documento chamado "Escolas Promotoras de Saúde: entorno saudável e meIhor saúde para as gerações futuras", com o intuito de divulgar conhecimentos e habilidades para o cuidado da própria saúde. ${ }^{8}$

Uma avaliação desse programa em Taiwan mostrou que, após as ações de educação adotadas, houve melhoria no conhecimento e habilidades para realizar o uso correto de medicamentos tais como: não comprar medicamentos sem prescrição médica, conferir informações na bula e seguir a adesão ao tratamento. ${ }^{2}$

Seguindo esse caminho de informação da população, o Ministério da Saúde lançou, em 2015, uma cartilha para a promoção do uso racional de medicamentos. ${ }^{9}$ Esta cartilha ilustra, de maneira didática, ações de promoção da saúde, informações sobre o momento da consulta com o médico, dispensação e orientação farmacêutica, armazenamento e descarte dos medicamentos.

Ainda é preciso, contudo, ampliar as ações que promovam o uso racional de medicamentos, incluindo aquelas direcionadas aos adolescentes, aproveitando o espaço da escola para promoção de saúde, mas também tentando alcançar as famílias e a comunidade. Para que estas estratégias sejam efetivas, faz-se necessário, inicialmente, conhecer o perfil de uso e de conhecimento sobre medicamentos desta população.

Editora Unijuí - Revista Contexto \& Saúde - ISSN 2176-7114 - v. 21, n. 44, out./dez. 2021 


\section{METODOLOGIA}

O estudo foi realizado com estudantes do Ensino Médio, modalidade regular e Educação de Jovens e Adultos (EJA), dos turnos diurno e noturno, regularmente matriculados em uma escola da rede pública estadual localizada na Região Metropolitana do Rio de Janeiro, com uma população de estudo de 800 alunos.

Usando como referência a porcentagem descrita na Pnaum, que aponta que $30,6 \%$ da população entre 10 e 19 anos faz uso de medicamentos, um erro amostral de $5 \%$ e um nível de confiança de 95\%, estabeleceu-se a amostra de 232 alunos.

Os dados foram coletados por meio de questionários semiestruturados autoaplicados, disponíveis on-line na ferramenta Google Forms, em computadores na sala de informática da escola. O questionário foi elaborado com base em outro trabalho semelhante, ${ }^{9}$ sendo dividido em dois blocos. O primeiro abordava questões sociodemográficas, consumo cotidiano de substâncias e acesso aos serviços de saúde, com perguntas sobre gênero, idade, turno e ano escolar, retenção, grau de escolaridade dos pais, renda familiar, trabalho, uso de tabaco, bebida alcoólica e drogas ilícitas. O segundo bloco apresentava perguntas sobre uso de medicamentos (regularmente e nos 15 dias anteriores), indicação, aquisição e orientação, frequência e motivos de automedicação e consultas com profissionais de saúde. Ao final, o aluno deveria escolher temas sobre uso de medicamentos a serem explorados em atividades escolares.

Previamente à data da aplicação dos questionários, o pesquisador explicou pessoalmente aos estudantes de todas as turmas o objetivo da pesquisa, convidando-os a participar de forma voluntária. Além disso, entregou o termo de consentimento para que os responsáveis pelos estudantes menores de 18 anos autorizassem sua inclusão no estudo, caso concordassem em participar.

A aplicação do questionário proposto foi realizada de junho a agosto de 2019, em datas especificadas pelo pesquisador, conforme o estabelecido pela direção pedagógica da unidade escolar. Nas datas preestabelecidas, os alunos foram acompanhados até a sala de informática para preencherem o questionário de maneira anônima e com as possíveis dúvidas respondidas pelo pesquisador. $\mathrm{O}$ preenchimento do questionário demorava cerca de 15 minutos. Foram incluídos os alunos menores de 18 anos que trouxeram o termo de consentimento dos responsáveis assinado, além de assinarem eles próprios um termo de assentimento. Os alunos maiores de 18 anos apenas precisaram assinar um termo de consentimento.

Para análise dos dados, todas as respostas foram exportadas para planiIhas do programa Microsoft Excel ${ }^{\circledR} 2013$. As variáveis sociodemográficas passaram por uma análise descritiva simples. $O$ uso de medicamentos foi analisado a partir da exata maneira como eles foram citados pelos participantes tomando por base segundo a Classificação Anatômica Química e Terapêutica (Anatomical Therapeutical Chemical, ATC). Tanto o uso regular quanto nos 15 dias anteriores à participação no estudo foram investigados. Para comparação entre diferentes grupos foi realizado o teste Qui-Quadrado, com a adoção de um nível de significância de $5 \%(p<0,05)$. 
Essa pesquisa foi aprovada pelo Comitê de Ética em Pesquisa (CEP) da Universidade Federal Fluminense (UFF) pelo parecer de número $3.302 .332 \mathrm{em}$ 3/5/2019 e obedeceu aos aspectos ético-legais vigentes da legislação brasileira para pesquisa com envolvimento de seres humanos.

\section{RESULTADOS}

\section{Caracterização da população de estudo}

Participaram da pesquisa 232 estudantes, todos matriculados no Ensino Médio, sendo 165 (71,12\%) do turno da manhã (modalidade regular). A maioria $(61,21 \%)$ pertencia ao gênero feminino. A idade ficou distribuída entre $14 \mathrm{e}$ mais de 20 anos, com o turno da manhã concentrando a maior parte dos alunos menores de 18 anos ( $n=107 ; 64,85 \%)$ e o turno da noite apresentando maior proporção de alunos maiores de 18 anos ( $n=67 ; 80,59 \%$ ).

Em relação à retenção escolar, o turno da manhã apresentou 96 alunos $(58,18 \%)$ que nunca haviam sido reprovados, enquanto no turno da noite 46 alunos $(68,66 \%)$ já haviam reprovado ao menos uma vez. O turno da noite concentrou maior parte dos alunos que exerciam alguma atividade remunerada $(n=34$; $50,75 \%)$ em relação aos alunos da manhã ( $n=16 ; 9,70 \%$ ).

A maioria dos estudantes dos dois turnos respondeu nunca ter feito uso de cigarros (manhã, $n=127 ; 76,97 \%$ e noite, $n=42 ; 62,69 \%$ ). No turno da manhã, a maior parte dos alunos $(n=82 ; 49,70 \%)$ respondeu ter usado uma vez algum tipo de bebida alcoólica, enquanto no turno noturno a maioria ( $n=22 ; 32,84 \%$ ) relatou ter usado de 6 a 20 vezes no último mês. Nos dois turnos, a maior parte dos alunos afirmou nunca ter consumido drogas ilícitas (manhã, $n=139 ; 84,24 \%$ e noite, $n=57 ; 85,97 \%)$.

Nos dois turnos a maioria afirmou ter ido a consultas médicas duas ou mais vezes no último ano (manhã, $n=106 ; 64,24 \%$ e noite, $n=41 ; 61,19 \%)$. O turno da noite, contudo, apresentou maior número de alunos $(n=53 ; 79,10 \%)$ se consultando com outros profissionais de saúde ( $n=94 ; 56,97 \%)$, com os mais citados sendo dentistas ( $n=82 ; 35,34 \%)$, nutricionistas $(n=24 ; 10,34 \%)$ e psicólogos $(n=23 ; 9,91 \%)$.

\section{Uso de medicamentos}

O uso de medicamentos foi avaliado em dois momentos: regular (todos os dias) e nos 15 dias anteriores à pesquisa. Além disso, avaliou-se a frequência e os motivos para automedicação. Não foi observada diferença estatisticamente significativa nessas variáveis entre os turnos.

O uso regular de medicamentos foi relatado por 74 alunos $(31,90 \%)$ e o uso nos 15 dias anteriores por 179 alunos (77,16\%). A maior parte dos alunos ( $n=93 ; 40,09 \%)$ afirmou quase nunca se automedicar, 76 (32,76\%) afirmaram se automedicar muitas vezes. Os principais motivos para essa prática envolveram alívio de dores ( $n=200,63,09 \%)$, alergia ( $n=55 ; 17,35 \%$ ) e alívio de tensões e estresse $(n=33 ; 10,41 \%)$. Nenhuma destas diferenças mostrou-se estatisticamente significativa (Tabela 1).

Editora Unijuí - Revista Contexto \& Saúde - ISSN 2176-7114 - v. 21, n. 44, out./dez. 2021 
Tabela 1 - Uso de medicamentos pelos estudantes, segundo o turno a que pertenciam, $\mathrm{N}=232$, Queimados, 2019

\begin{tabular}{|c|c|c|c|c|c|c|c|c|}
\hline \multirow{2}{*}{ Uso de medicamentos } & $(\mathrm{N}=165)$ & $\%$ & $(\mathrm{~N}=67)$ & $\%$ & $(\mathrm{~N}=232)$ & $\%$ & \multirow{2}{*}{$\mathrm{p}$} \\
\cline { 2 - 7 } & \multicolumn{2}{|c|}{ Manhã } & \multicolumn{2}{|c|}{ Noite } & \multicolumn{2}{|c|}{ Total } & \\
\hline Uso Regular & & & & & & & 0,844 \\
\hline Sim & 52 & 31,52 & 22 & 32,84 & 74 & 31,90 & \\
\hline Não & 113 & 68,48 & 45 & 67,16 & 158 & 68,10 & \\
\hline Uso nos 15 dias anteriores & & & & & & & 0,202 \\
\hline Sim & 131 & 79,39 & 48 & 71,64 & 179 & 77,16 & \\
\hline Não & 34 & 20,61 & 19 & 28,36 & 53 & 22,84 & \\
\hline Automedicação & & & & & & & 0,549 \\
\hline Nunca & 13 & 7,88 & 9 & 13,43 & 22 & 9,48 & \\
\hline Quase Nunca & 66 & 40,00 & 27 & 40,30 & 93 & 40,09 & \\
\hline Muitas Vezes & 57 & 34,55 & 19 & 28,36 & 76 & 32,76 & \\
\hline Sempre & 29 & 17,58 & 12 & 17,91 & 41 & 17,67 & \\
\hline Motivos para automedicação & & & & & & & 0,489 \\
\hline Alívio de dores & 145 & 61,44 & 55 & 67,90 & 200 & 63,09 & \\
\hline Alergia & 41 & 17,37 & 14 & 17,28 & 55 & 17,35 & \\
\hline Alívio de tensões e estresses & 26 & 11,02 & 7 & 8,64 & 33 & 10,41 & \\
\hline Combate a doenças & 21 & 8,90 & 3 & 3,70 & 24 & 7,57 & \\
\hline Outros & 3 & 1,27 & 2 & 2,47 & 5 & 1,58 & \\
\hline
\end{tabular}

\%: Porcentual, p: nível de significância. Teste Qui-Quadrado

Fonte: Dados da pesquisa.

Um total de 142 especialidades farmacêuticas foram citados como medicamentos de uso regular, sendo a dipirona o mais mencionado ( $n=30 ; 21,13 \%)$, seguido da composição dipirona + orfenadrina + cafeína ( $n=19 ; 13,38 \%)$ e anticoncepcionais ( $n=17 ; 11,97 \%$ ). O Gráfico 1 mostra a distribuição dos 10 produtos mais referidos.

Gráfico 1 - Distribuição dos 10 medicamentos mais utilizados por uso regular, $\mathrm{N}=142$, Queimados, 2019

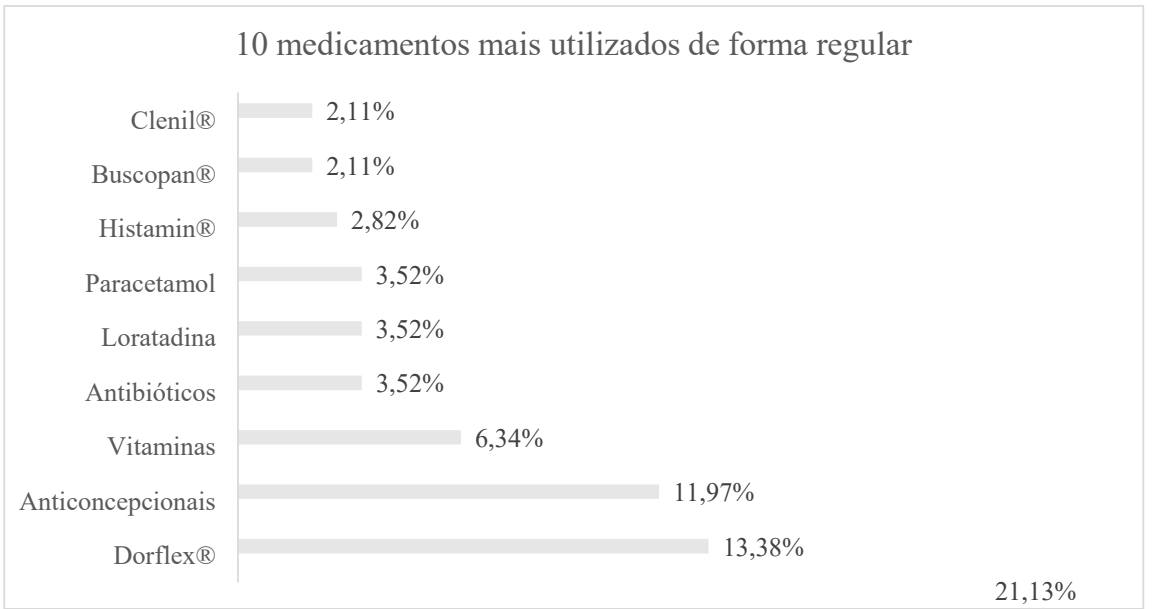

Legenda: Clenil ${ }^{\circledR}$ (Dipropionato de beclometasona); Buscopan ${ }^{\circledR}$ (Escopolamina); Histamin ${ }^{\circledR}$ (Dexclorfeniramina) e Dorflex ${ }^{\circledR}$ (dipirona + orfenadrina + cafeína).

Fonte: Dados da pesquisa. 
Entre os alunos que afirmaram usar medicamentos regularmente, 58 $(79,45 \%)$ disseram ter sido orientados. O principal responsável por esta orientação foi o médico ( $n=50,55,56 \%)$, seguido de algum familiar ( $n=21 ; 23,33 \%)$ e do farmacêutico ( $n=9 ; 10,00 \%)$. A maior parte dos alunos ( $n=67 ; 91,78 \%)$ obteve o medicamento em alguma farmácia privada.

Foi observada associação significativa entre o uso regular de medicamentos e o gênero feminino ( $n=61 ; 82,43 \% ; p<0,001)$, a ida a duas ou mais consultas médicas no último ano $(n=57 ; 77,03 \% ; p=0,003)$ e a realização de consulta com outro profissional de saúde ( $n=61 ; 82,43 \% ; p<0,001)$ (Tabela 2).

As alunas em uso de anticoncepcionais representaram apenas cerca de $12 \%$ da amostra. A maior parte das alunas em uso destes produtos tinha menos de 18 anos (53\%), pertencia ao turno da noite (53\%), não trabalhava (64\%), tinha ido a duas ou mais consultas médicas no último ano (70\%) e receberam orientação médica para uso (94\%).

Tabela 2 - Variáveis relacionadas ao uso medicamentos de maneira regular e nos 15 dias anteriores à pesquisa pelos estudantes, $N=232$, Queimados, 2019

\begin{tabular}{|c|c|c|c|c|c|c|c|c|c|c|}
\hline \multirow[b]{2}{*}{ Variáveis } & \multicolumn{4}{|c|}{ Uso regular } & \multirow[b]{2}{*}{$p$} & \multicolumn{4}{|c|}{ Uso nos 15 dias anteriores } & \multirow[b]{2}{*}{$p$} \\
\hline & $\operatorname{Sim}_{(n=74)}$ & $\%$ & $\begin{array}{c}\text { Não } \\
(n=158)\end{array}$ & $\%$ & & $\operatorname{Sim}_{(n=179)}$ & $\%$ & $\begin{array}{c}\text { Não } \\
(n=53)\end{array}$ & $\%$ & \\
\hline ênero & & & & & $<0,001$ & & & & & 0,006 \\
\hline Masculino & 13 & 17,57 & 77 & 48,73 & & 61 & 34,08 & 29 & 54,72 & \\
\hline Feminino & 61 & 82,43 & 81 & 51,27 & & 118 & 65,92 & 24 & 45,28 & \\
\hline Idade & & & & & 0,950 & & & & & 0,330 \\
\hline Menos de 14 & 1 & 1,35 & 1 & 0,63 & & 1 & 0,56 & 1 & 1,89 & \\
\hline 15 & 8 & 10,81 & 12 & 7,59 & & 13 & 7,26 & 7 & 13,21 & \\
\hline 16 & 15 & 20,27 & 35 & 22,15 & & 43 & 24,02 & 7 & 13,21 & \\
\hline 17 & 14 & 18,92 & 34 & 21,52 & & 34 & 18,99 & 14 & 26,42 & \\
\hline 18 & 20 & 27,03 & 41 & 25,95 & & 50 & 27,93 & 11 & 20,75 & \\
\hline 19 & 8 & 10,81 & 14 & 8,86 & & 16 & 8,94 & 6 & 11,32 & \\
\hline Mais de 20 & 8 & 10,81 & 21 & 13,29 & & 22 & 12,29 & 7 & 13,21 & \\
\hline $\begin{array}{c}\text { Retenção } \\
\text { escolar }\end{array}$ & & & & & 0,099 & & & & & 0,923 \\
\hline Não & 35 & 47,30 & 82 & 51,90 & & 89 & 49,72 & 28 & 52,83 & \\
\hline Sim- 1 vez & 30 & 40,54 & 44 & 27,85 & & 58 & 32,40 & 16 & 30,19 & \\
\hline im- 2 vezes & 9 & 12,16 & 32 & 20,25 & & 32 & 17,88 & 9 & 16,98 & \\
\hline $\begin{array}{l}\text { scolaridade } \\
\text { haterna }\end{array}$ & & & & & 0,686 & & & & & 0,396 \\
\hline $\begin{array}{c}\text { Nunca estu- } \\
\text { dou }\end{array}$ & 1 & 1,35 & 1 & 0,63 & & 2 & 1,12 & 0 & 0,00 & \\
\hline Até $5^{\circ} \mathrm{EF}$ & 20 & 27,03 & 32 & 20,25 & & 37 & 20,67 & 15 & 28,30 & \\
\hline Até $9^{\circ} \mathrm{EF}$ & 10 & 13,51 & 33 & 20,89 & & 30 & 16,76 & 13 & 24,53 & \\
\hline Até EM & 23 & 31,08 & 46 & 29,11 & & 58 & 32,40 & 11 & 20,75 & \\
\hline Até ES & 9 & 12,16 & 23 & 14,56 & & 26 & 14,53 & 6 & 11,32 & \\
\hline $\begin{array}{l}\text { Jão há infor- } \\
\text { mação }\end{array}$ & 11 & 14,86 & 23 & 14,56 & & 26 & 14,53 & 8 & 15,09 & \\
\hline $\begin{array}{l}\text { scolaridade } \\
\text { aterna }\end{array}$ & & & & & 0,511 & & & & & 0,988 \\
\hline unca estudou & 4 & 5,41 & 4 & 2,53 & & 6 & 3,35 & 2 & 3,77 & \\
\hline Até $5^{\circ} \mathrm{EF}$ & 18 & 24,32 & 28 & 17,72 & & 34 & 18,99 & 12 & 22,64 & \\
\hline Até $9^{\circ} \mathrm{EF}$ & 9 & 12,16 & 26 & 16,46 & & 28 & 15,64 & 7 & 13,21 & \\
\hline
\end{tabular}




\begin{tabular}{|c|c|c|c|c|c|c|c|c|c|c|}
\hline Até EM & 17 & 22,97 & 35 & 22,15 & & 41 & 22,91 & 11 & 20,75 & \\
\hline Até ES & 3 & 4,05 & 13 & 8,23 & & 12 & 6,70 & 4 & 7,55 & \\
\hline $\begin{array}{l}\text { Não há infor- } \\
\text { mação }\end{array}$ & 23 & 31,08 & 52 & 32,91 & & 58 & 32,40 & 17 & 32,08 & \\
\hline $\begin{array}{l}\text { Renda fami- } \\
\text { liar (em SM) }\end{array}$ & & & & & 0,217 & & & & & 0,849 \\
\hline Menos de 3 & 52 & 70,27 & 111 & 70,25 & & 125 & 69,83 & 38 & 71,70 & \\
\hline De 3 a 5 & 17 & 22,97 & 39 & 24,68 & & 44 & 24,58 & 12 & 22,64 & \\
\hline De 5 a 10 & 3 & 4,05 & 8 & 5,06 & & 8 & 4,47 & 3 & 5,66 & \\
\hline Mais de 10 & 2 & 2,70 & 0 & 0,00 & & 2 & 1,12 & 0 & 0,00 & \\
\hline $\begin{array}{l}\text { Atividade } \\
\text { remunerada }\end{array}$ & & & & & 0,296 & & & & & 0,826 \\
\hline $\operatorname{sim}$ & 19 & 25,68 & 31 & 19,62 & & 38 & 21,23 & 12 & 22,64 & \\
\hline Não & 55 & 74,32 & 127 & 80,38 & & 141 & 78,77 & 41 & 77,36 & \\
\hline Uso de cigarro & & & & & 0,633 & & & & & 0,465 \\
\hline Nunca usei & 56 & 75,68 & 113 & 71,52 & & 128 & 71,51 & 41 & 77,36 & \\
\hline Uma vez & 14 & 18,92 & 33 & 20,89 & & 39 & 21,79 & 8 & 15,09 & \\
\hline 6 a 20 vezes & 1 & 1,35 & 7 & 4,43 & & 5 & 2,79 & 3 & 5,66 & \\
\hline $\begin{array}{c}\text { Mais de } 20 \\
\text { vezes }\end{array}$ & 3 & 4,05 & 5 & 3,16 & & 7 & 3,91 & 1 & 1,89 & \\
\hline $\begin{array}{l}\text { Uso de bebi- } \\
\text { das alcoólicas }\end{array}$ & & & & & 0,886 & & & & & 0,620 \\
\hline Nunca usei & 19 & 25,68 & 47 & 29,75 & & 49 & 27,37 & 17 & 32,08 & \\
\hline Uma vez & 33 & 44,59 & 68 & 43,04 & & 78 & 43,58 & 23 & 43,40 & \\
\hline 6 a 20 vezes & 17 & 22,97 & 31 & 19,62 & & 40 & 22,35 & 8 & 15,09 & \\
\hline $\begin{array}{c}\text { Mais de } 20 \\
\text { vezes }\end{array}$ & 5 & 6,76 & 112 & 7,59 & & 12 & 6,70 & 5 & 9,43 & \\
\hline $\begin{array}{l}\text { Uso de dro- } \\
\text { gas ilícitas }\end{array}$ & & & & & 0,435 & & & & & 0,935 \\
\hline Nunca usei & 64 & 86,49 & 132 & 83,54 & & 151 & 84,36 & 45 & 84,91 & \\
\hline Uma vez & 4 & 5,41 & 18 & 11,39 & & 17 & 9,50 & 5 & 9,43 & \\
\hline 6 a 20 vezes & 3 & 4,05 & 4 & 2,53 & & 5 & 2,79 & 2 & 3,77 & \\
\hline $\begin{array}{c}\text { Mais de } 20 \\
\text { vezes }\end{array}$ & 3 & 4,05 & 4 & 2,53 & & 6 & 3,35 & 1 & 1,89 & \\
\hline $\begin{array}{l}\text { Consultas } \\
\text { médicas no } \\
\text { último ano } \\
\end{array}$ & & & & & 0,003 & & & & & 0,019 \\
\hline Nenhuma & 2 & 2,70 & 24 & 15,19 & & 18 & 10,06 & 8 & 15,09 & \\
\hline Uma vez & 15 & 20,27 & 44 & 27,85 & & 39 & 21,79 & 20 & 37,74 & \\
\hline $\begin{array}{c}\text { Duas vezes } \\
\text { ou mais }\end{array}$ & 57 & 77,03 & 90 & 56,96 & & 122 & 68,16 & 25 & 47,17 & \\
\hline $\begin{array}{c}\text { Consultas a ou- } \\
\text { tros profissionais } \\
\text { de saúde }\end{array}$ & & & & & $<0,001$ & & & & & 0,001 \\
\hline Sim & 61 & 82,43 & 86 & 54,43 & & 123 & 68,72 & 24 & 45,28 & \\
\hline Não & 13 & 17,57 & 72 & 45,57 & & 56 & 31,28 & 29 & 54,72 & \\
\hline $\begin{array}{l}\text { Automedica- } \\
\text { ção }\end{array}$ & & & & & 0,404 & & & & & $<0,001$ \\
\hline Nunca & 4 & 5,41 & 18 & 11,39 & & 12 & 6,70 & 10 & 18,87 & \\
\hline Quase Nunca & 29 & 39,19 & 64 & 40,51 & & 62 & 34,64 & 31 & 58,49 & \\
\hline Muitas Vezes & 25 & 33,78 & 51 & 32,28 & & 65 & 36,31 & 11 & 20,75 & \\
\hline Sempre & 16 & 21,62 & 25 & 15,82 & & 40 & 22,35 & 1 & 1,89 & \\
\hline
\end{tabular}

EM: Ensino Médio; EF: Ensino Fundamental; ES: Ensino Superior; SM: Salários Mínimos; \%: Porcentual, p: nível de significância.

Teste Qui Quadrado 
Os dados sobre o uso de medicamentos nos 15 dias anteriores à pesquisa mostraram o consumo de 237 especialidades farmacêuticas, com os medicamentos mais usados sendo os mesmos apontados no uso regular (Gráfico 2). A indicação de um profissional de saúde para o uso desses medicamentos foi confirmada por 95 alunos (55,88\%), sendo os maiores responsáveis pela indicação de uso os médicos ( $n=77 ; 38,12 \%)$, seguidos dos farmacêuticos ( $n=68 \% ; 33,66 \%)$. A indicação por algum familiar foi citada por 29 estudantes (14,36\%) e as farmácias privadas foram os locais de aquisição apontados pela maioria ( $n=155 ; 92,81 \%)$.

Houve associação significativa entre o perfil de uso e o gênero $(p=0,006)$, com o gênero feminino se destacando ( $n=118 ; 65,92 \%)$, número de consultas médicas no último ano $(p=0,019)$, consultas a outros profissionais de saúde $(p=0,001)$ e frequência de automedicação $(p<0,001)$. (Tabela 2$)$.

Gráfico 2 - Distribuição dos 10 medicamentos mais utilizados 15 dias antes da pesquisa, $\mathrm{N}=237$, Queimados, 2019

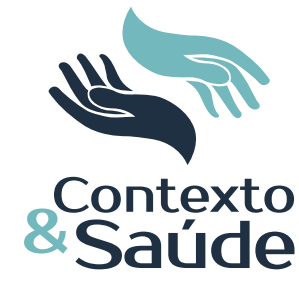

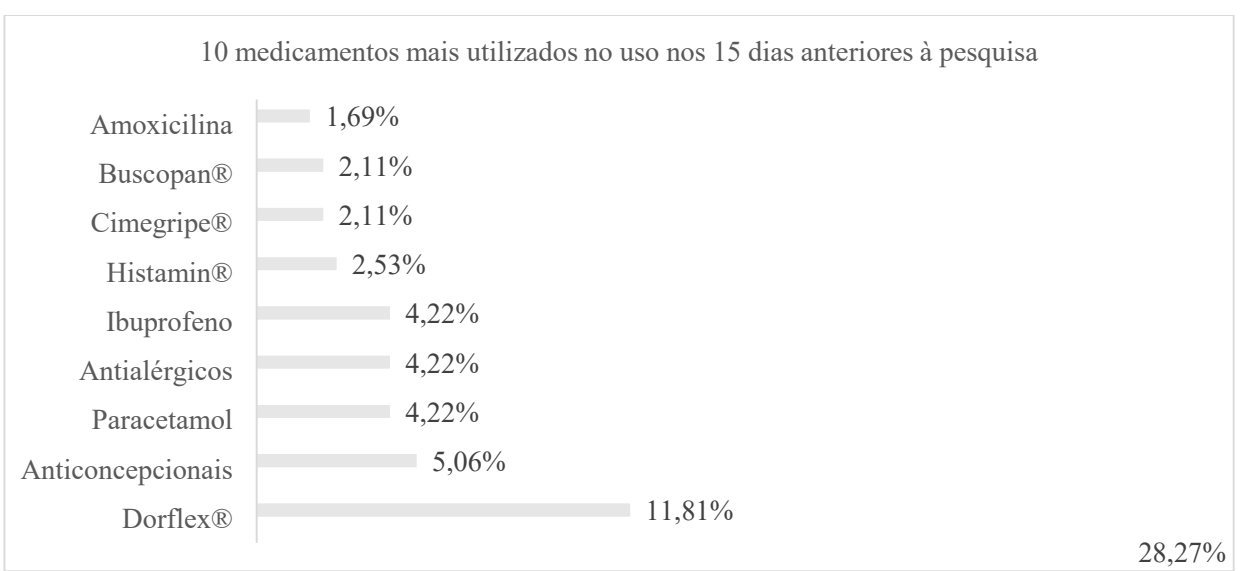

Legenda: Buscopan ${ }^{\circledR}$ (Escopolamina); Cimegripe ${ }^{\circledR}($ paracetamol + maleato de clorniferamina + cloridrato de fenilefrina) e Dorflex® (dipirona + orfenadrina + cafeína).

Fonte: Dados da pesquisa.

\section{Comparação Uso Regular e Uso nos 15 dias anteriores}

O uso de medicamentos nos últimos 15 dias $(77,16 \%)$ mostrou-se superior ao uso regular $(31,90 \%)(p<0,001)$. O perfil de medicamentos usados foi semeIhante nas duas situações de uso, com pequenas diferenças percentuais entre os grupos de medicamentos usados, que não se mostraram estatisticamente significativas $(p=0,170)$. Os medicamentos com atuação no sistema nervoso central foram os mais consumidos nos dois casos, com destaque para os analgésicos $(26,76 \%)$, seguidos pelos medicamentos de ação no sistema respiratório (20,42\%), em que se destacaram os anti-histamínicos (11,27\%).

Foi verificada associação significativa entre acesso à orientação médica e uso de medicamentos por indicação $(p<0,001)$, que se mostrou mais frequente para os medicamentos de uso regular ( $n=58 ; 79 ; 45 \%)$. A indicação para uso de medicamentos pelo farmacêutico, por outro lado, foi mais comum para os medicamentos de uso nos 15 dias anteriores à pesquisa ( $n=68 ; 33,66 \%)$ (Tabela 3). 
Tabela 3 - Comparação entre o uso de medicamentos regular

e nos 15 dias anteriores à pesquisa, Queimados, N=232, 2019

\begin{tabular}{|c|c|c|c|c|c|}
\hline \multirow{2}{*}{ Uso de medicamentos } & \multicolumn{2}{|c|}{ Regular } & \multicolumn{2}{|c|}{15 dias anteriores } & \multirow{2}{*}{$\mathrm{p}$} \\
\hline & $\mathrm{N}$ & $\%$ & $\mathrm{~N}$ & $\%$ & \\
\hline Uso & & & & & $<0,001$ \\
\hline $\operatorname{sim}$ & 74 & 31,90 & 179 & 77,16 & \\
\hline Não & 158 & 68,10 & 53 & 22,84 & \\
\hline Orientação para uso & & & & & $<0,001$ \\
\hline $\operatorname{sim}$ & 58 & 79,45 & 95 & 55,88 & \\
\hline Não & 15 & 20,55 & 75 & 44,12 & \\
\hline Indicação de uso & & & & & $<0,001$ \\
\hline Familiar & 21 & 23,33 & 29 & 14,36 & \\
\hline Balconista & 2 & 2,22 & 19 & 9,41 & \\
\hline Propaganda & 2 & 2,22 & 0 & 0,00 & \\
\hline Médico & 50 & 55,56 & 77 & 38,12 & \\
\hline Receita antiga & 2 & 2,22 & 6 & 2,97 & \\
\hline Farmacêutico & 9 & 10,00 & 68 & 33,66 & \\
\hline Outros & 4 & 4,44 & 3 & 1,49 & \\
\hline Local de compra & & & & & 0,779 \\
\hline Rede privada & 67 & 91,78 & 155 & 92,81 & \\
\hline Rede pública & 6 & 8,22 & 12 & 7,19 & \\
\hline Automedicação & & & & & 0,908 \\
\hline Nunca & 4 & 5,41 & 12 & 6,70 & \\
\hline Quase Nunca & 29 & 39,19 & 62 & 34,64 & \\
\hline Muitas vezes & 25 & 33,78 & 65 & 36,31 & \\
\hline Sempre & 16 & 21,62 & 40 & 22,35 & \\
\hline
\end{tabular}

\%: Porcentual, p: nível de significância.

Teste Qui-Quadrado

Fonte: Dados da pesquisa.

Entre os temas apontados pelos estudantes como de interesse para atividades escolares se destacaram os riscos da automedicação ( $n=123 ; 31,87 \%)$, uso e controle de anticoncepcionais $(n=58 ; 15,03 \%)$ e uso de medicamentos para controle de peso $(n=57 ; 14,77 \%)$.

\section{Discussão}

O perfil de consumo regular de medicamentos está de acordo com os resultados da Pnaum, ${ }^{6}$ que apontam que cerca de $31 \%$ da população entre 10 e 19 anos faz uso regular de medicamentos. É inferior, no entanto, ao observado por outros autores que relatam uso regular de medicamentos por $52 \%$ dos mais de 400 alunos entrevistados. ${ }^{11}$ Abrahão et al. , num universo de mais de 1000 estudantes, encontrou o uso regular em $12 \%$ deles $^{10}$. O uso de medicamentos nos 15 dias anteriores à pesquisa foi superior ao observado em outros estudos, que apontaram valores na faixa de $36 \%$ a $56,6 \%$ na semana anterior à coleta de dados. 8,12

A frequência de automedicação observada está de acordo com outras pesquisas que observaram automedicação em percentual superior a $50 \%$ dos estudantes entrevistados. ${ }^{5,12}$ 
Embora a Pnaum tenha identificado automedicação em apenas $16,1 \%$ da população brasileira, ${ }^{13}$ a pesquisa apontou associação positiva para sexo feminino e as faixas etárias de 10 a 19 anos. Essa observação é coerente com o fato de a automedicação ter sido o tema de maior interesse para palestras sobre uso de medicamentos.

Estudo feito com mais de 6 mil alunos de todo o ciclo básico de educação em Taiwan mostrou associação entre o uso de álcool e tabaco com a automedicação, ${ }^{14}$ resultado que não foi observado neste estudo.

Entre as razões apontadas para a automedicação destacaram-se o alívio de dores e alergia, o que é coerente tanto com o perfil de medicamentos usados quanto com os resultados de outros estudos. ${ }^{5,15}$ Uma revisão sistemática feita em 163 estudos sobre automedicação entre adolescentes de 13 a 18 anos também identificou dor de cabeça e muscular, alergia e febre como as principais causas para automedicação. ${ }^{16}$

A maior utilização de medicamentos para tratamento da dor e para doenças do trato respiratório, tanto como uso crônico quanto nos 15 dias anteriores à pesquisa, mostrou-se semelhante ao observado em outros estudos com estudantes dessa faixa etária. ${ }^{5,12,13}$ Outro aspecto que pode influenciar o maior uso de analgésicos nos 15 dias anteriores à pesquisa é que parte destes produtos são MIP (medicamento isento de prescrição) e apresentam baixo custo, tendo, por isso, o consumo facilitado. ${ }^{15}$

Apesar de questionados sobre uso de medicamentos para dificuldade de aprendizagem, apenas 1 aluno $(0,43 \%)$ fez menção ao uso de cloridrato de metilfenidato. Este resultado difere do observado por Almeida et al. ${ }^{11}$ que relataram maior frequência de uso desta substância entre alunos de Ensino Médio de duas escolas no sul do Brasil. Raman et al., ${ }^{17}$ em estudo retrospectivo em países na Ásia, Austrália, América do Norte e Europa, observaram prevalência crescente do uso de metilfenidato entre crianças e adolescentes, com variação de 0,27 a 6,69\% entre os diferentes países. Mac Avin et al. ${ }^{18,}$ ao analisarem os padrões de prescrição do metilfenidato e outros medicamentos psicotrópicos para tratamento do déficit de atenção em população com menos de 25 anos na Irlanda, no período de 2005 a 2015, observaram níveis maiores de prescrição de metilfenidato em 2015, assim como aumento na prescrição concomitante de antidepressivos. Da mesma forma, a baixa frequência de medicamentos para ansiedade e depressão difere dos resultados observados por outros estudos. Kovess et al. ${ }^{19,}$ ao analisarem o perfil de uso de medicamentos por mais de 120 mil crianças e adolescentes, encontraram que 2,5\% fazia uso de algum psicotrópico, número similar ao encontrado em estudos holandeses e menor do que estudos norte-americanos. Um estudo feito com mais de 5 mil estudantes na região sul do Brasil mostrou que $7,7 \%$ dos alunos entrevistados já tinham usado algum ansiolítico e $1,1 \%$ fizeram uso de barbitúricos em algum momento da vida. ${ }^{4}$

O gênero mostrou associação significativa com o uso de medicamentos, tanto regular quanto recente, com predominância de uso entre as mulheres. Isto pode ser explicado tanto pelo uso de anticoncepcionais quanto pela maior procura por serviços de saúde e maiores cuidados com a saúde entre as mulheres, como aponta a literatura. ${ }^{13,14}$ 
Estudo feito com adolescentes do Ensino Médio de uma escola pública do Maranhão mostrou que cerca de $25 \%$ das jovens já haviam iniciado sua vida sexual e faziam uso de pílula contraceptiva. ${ }^{20}$ Borges et al. ${ }^{21}$, ao analisarem uma amostra nacional de estudantes brasileiros encontrou prevalência de $24,7 \%$ do uso de pílula anticoncepcional na última relação sexual, principalmente entre as adolescentes mais velhas (20,8\%). Ambos os resultados são superiores ao observado neste estudo. Isto leva ao questionamento da possibilidade de sonegação da informação sobre o uso destes medicamentos, o que poderia estar relacionado à automedicação. Isto seria coerente com o fato de que, entre os temas apontados pelos jovens como de interesse para obter mais informações, o uso e controle de anticoncepcionais foi o segundo mais votado, com o interesse maior sendo entre as meninas (69,89\%). Por outro lado, Chofakian et al., ${ }^{22}$ em pesquisa com estudantes de São Paulo entre 18 e 24 anos, observaram dos 67\% de mulheres que usavam contraceptivos orais, $19 \%$ já haviam descontinuado o uso destes produtos em razão de eventos adversos.

Almeida et al. ${ }^{11}$ chamaram a atenção, entretanto, para a possibilidade de o uso regular de anticoncepcionais ser feito sem prescrição. Segundo os autores, isto poderia ser explicado pela aquisição inicial do produto com uma prescrição e, posteriormente, a continuidade do uso por automedicação, sem uma nova prescrição ou reavaliação. Além disso, Zanini et al. ${ }^{23}$ consideram que o uso inadequado destes produtos possa ser uma das causas de gravidez indesejada entre os adolescentes, aspecto também ressaltado por Chofakian et al. ${ }^{22}$

O consumo de medicamentos por adolescentes também está diretamente ligado à influência do ambiente familiar. Shehnaz et al. ${ }^{16}$ identificaram os pais como os principais protagonistas na recomendação, indicação e informação sobre medicamentos. Mais especificamente, alguns estudos mostraram que as mães são as principais indutoras do uso de medicamentos, o que se relaciona também com seu nível de escolaridade. ${ }^{10,12}$ As mães de maior nível de escolaridade apresentam um maior consumo regular de medicamentos, influenciando seus filhos. É razoável supor que quanto maior a escolaridade, maior o acesso à informação e aos serviços de saúde, assim como à automedicação. Um estudo feito na Noruega com adolescentes e suas mães mostrou associação entre a relação materna e o controle de dor, incluindo o uso de MIP. ${ }^{24}$

Júnior e Silva ${ }^{4}$ identificaram que o farmacêutico deveria ser o profissional mais adequado para promover a educação em saúde e o uso correto de medicamentos. Neste estudo, entretanto, um baixo percentual de alunos foi orientado pelo farmacêutico em relação ao uso dos medicamentos. Além disso, a indicação de uso pelo farmacêutico foi maior no uso de medicamentos nos 15 dias anteriores à pesquisa, sugerindo que o profissional esteja sendo mais procurado no processo de escolha de MIP ou de automedicação.

Este estudo observou que a aquisição de medicamentos foi maior em farmácias privadas. Pode-se explicar pela facilidade do acesso a esses produtos nestes estabelecimentos, inclusive em razão do alto número de drogarias e farmácias privadas e pelas limitações de acesso aos serviços de saúde.

Neste cenário, é importante destacar a postura do farmacêutico em ações que promovam o uso racional de medicamentos nestes estabelecimentos. Pes- 
quisa recente realizada com adolescentes portugueses sobre cuidados em saúde mostrou que, entre os profissionais a que recorrem, o farmacêutico estava em terceiro lugar (18,2\%), sendo citados em relação à eficiência, informação e esclarecimento. ${ }^{25}$

Da mesma forma, Paim et al. ${ }^{15}$ chamaram a atenção para a necessidade de todos os profissionais de saúde e educadores em saúde alertarem a população sobre os riscos e dúvidas da prática da automedicação. Com isso, a escola torna-se ambiente propício para ações de saúde direcionadas a crianças e adolescentes.

\section{CONCLUSÃO}

Embora a resposta ao instrumento tenha sido realizada pelo próprio aluno e o local de pesquisa tenha se restringido a uma única escola, principais limitações deste estudo, foi possível observar o perfil de consumo de medicamentos pelos adolescentes. Apesar de se ter buscado assegurar privacidade na resposta e o tempo necessário para a finalização do processo, é possível que aspectos como o consumo de tabaco, álcool, drogas ilícitas e anticoncepcionais possam ter sido subestimados por constrangimento na resposta aos questionários.

A escola como ambiente de formação, tendo a saúde como tema transversal a ser trabalhado de maneira multidisciplinar, pode funcionar como espaço de promoção da saúde, ainda mais levando-se em conta a questão da automedicação como prática crescente, principalmente em adolescentes, devido a dificuldades de acesso aos serviços de saúde.

Os resultados indicam que é necessário falar sobre o uso de medicamentos dentro do espaço escolar, quer seja pela relevância do tema para a saúde pública quer seja pelo interesse por parte dos próprios alunos. Nesse sentido, a inserção dos profissionais de saúde, seja na capacitação de professores ou no processo de oferecimento de informações sobre medicamentos nas escolas, apresenta grande potencial na promoção do uso racional de medicamentos.

A escola como espaço de promoção da saúde tem grande potencial de contribuição na promoção do uso racional de medicamentos, sobretudo em razão da alta taxa de automedicação e de intoxicação por medicamentos entre os adolescentes. Além de relevante, o tema representa um aspecto de interesse dos estudantes.

\section{REFERÊNCIAS}

${ }^{1}$ Sistema Nacional de Informações Tóxico-Farmacológicas (SNITF). 2019. Dados de intoxicação. [Acesso em: 15 mar. 2019]. Disponível em: http://sinitox.icict.fiocruz.br/dados-nacionais.

${ }^{2} \mathrm{Chi} \mathrm{H}$ et al. Evaluation of a health-promoting school program to enhance correct medication use in Taiwan, Journal of Food and Drug Analysis, Taiwan. 2014;22(2):271-78.

${ }^{3}$ Pizzol TSD et al. Uso de medicamentos e outros produtos com finalidade terapêutica entre crianças no Brasil. Rev. de Saúde Pública, São Paulo. 2016;50, supl. 2, 5s.

${ }^{4}$ Junior NMC, Silva, JRS. (In)Visibilidade da Escola na Discussão Sobre o Uso Racional de Medicamentos. Contexto \& Educação, Ijuí: Unijuí. 2017;32(102):145-69. 
${ }^{5}$ Matos JF et al. Prevalência, perfil e fatores associados à automedicação em adolescentes e servidores de uma escola pública profissionalizante. Cad. Saúde Coletiva. 2018;26(1):76-83.

${ }^{6}$ Bertoldi AD et al. Perfil sociodemográfico dos usuários de medicamentos no Brasil: resultados da PNAUM 2014. Rev. de Saúde Pública, São Paulo. 2016;50, supl. 2, 5 s.

${ }^{7}$ Bermudez JAZ, Barros MBA. Perfil do acesso e da utilização de medicamentos da população brasileira - contribuições e desafios da PNAUM - Inquérito Domiciliar. Rev. Saúde Pública, São Paulo. 2016;50, supl. 2, 2s.

${ }^{8}$ Organização Pan-Americana de Saúde (Opas). Red Latinoamericana de escuelas promotoras de la salud. Washigton, DC. 1998.

${ }^{9}$ Brasil. Ministério da Saúde. Cartilha para a promoção do uso racional de medicamentos. Brasília. 2015.

${ }^{10}$ Abrahão RC, Godoy JA, Halpern R. Automedicação e comportamento entre adolescentes em uma cidade do Rio Grande do Sul. Aletheia, Canoas. 2013;41:134-53.

${ }^{11}$ Almeida $C$ et al. Levantamento do uso de medicamentos por estudantes do Ensino Médio em duas escolas de Porto Alegre, RS, Brasil. Ciência \& Educação, Bauru. 2012;18(1):215-30.

12 Pereira FSVT et al. Automedicação em crianças e adolescentes. Jornal de Pediatria. 2007;83(5):453-58.

${ }^{13}$ Arrais PSD et al. Prevalência da automedicação no Brasil e fatores associados. Rev. Saúde Pública, São Paulo. 2016;50, supl. 2, 13s.

${ }^{14}$ Lee $\mathrm{C}$ et al. Inappropriate self-medication among adolescents and its association with lower medication literacy and substance use. Plos One. 2017;12(12):e0189199.

${ }^{15}$ Paim RSP et al. Automedicação: uma síntese das publicações nacionais. Rev. Contexto \& Saúde, ljuí: Editora Unijuí. 2016;16(30):47-54.

${ }^{16}$ Shehnaz SI et al. A systematic review of self-medication practices among adolescents. The Journal of Adolescent Health. 2014;55(4):467-83.

${ }^{17}$ Raman SR et al. Trends in attention-deficit hyperactivity disorder medication use: a retrospective observational study using population-based databases. Lancet Psychiatry. 2018;5(10):824-35.

${ }^{18} \mathrm{Mac}$ Avin $\mathrm{M}$ et al. Trends in attention deficit and hyperactivity disorder (ADHD) medications among children and young adults in Ireland: a repeated cross-sectional study from 2005 to 2015. BMJ Open. 2020;10(4).

${ }^{19}$ Kovess $V$ et al. Psychotropic medication use in French children and adolescents. Journal of Child and Adolescent Psychopharmacology, New York. 2015;25(2):168-75.

${ }^{20}$ Ramos LA et al. Uso de métodos anticoncepcionais por mulheres adolescentes de escola pública. Cogitare Enfermagem. 2018;23(3).

${ }^{21}$ Borges ALV et al. Erica: sexual initiation and contraception in Brazilian adolescents. Rev. de Saúde Pública, São Paulo. 2016;50, supl. 1.

${ }^{22}$ Chofakian CBN, Moreau C, Borges ALV et al. Contraceptive discontinuation: frequency and associated factors among undergraduate women in Brazil. Reprod Health. 2019;16(131).

${ }^{23}$ Zanini $\mathrm{M}$ et al. Uso de contraceptivos e fatores associados entre adolescentes de 15 a 18 anos de idade em Unidade de Saúde da Família. Revista de Medicina. 2017;96(1).

${ }^{24}$ Skarstein $S$ et al. Pain and development of identity in adolescents who frequently use over-the-counter analgesics: a qualitative study. Journal of Clinical Nursing. 2018;27(19-20):3583-91.

${ }^{25}$ Vinagre MG, Barros L. Preferências dos adolescentes sobre os cuidados de saúde. Ciênc. Saúde Coletiva. 2019;24(5):1627-36. 